Check for updates

Cite this as: $B M J 2021 ; 374: n 2152$ http://dx.doi.org/10.1136/bmj.n2152 Published: 01 September 2021

\section{Covid-19: Delta variant doubles risk of hospital admission compared with alpha variant, study shows}

\author{
Jacqui Wise
}

Patients with the delta variant of SARS-CoV-2 have more than double the risk of hospital admission compared with those infected with the alpha variant, according to a large UK study. ${ }^{1}$

The research, published in the Lancet Infectious Diseases, is the first to report hospital admission risk for the delta compared with the alpha variants based on cases confirmed by whole genome sequencing.

Researchers analysed healthcare data from 43338 positive covid-19 cases in England between 29 March and 23 May 2021. During the study period, 8682 patients were infected with the delta variant and 34 656 with the alpha variant. Although the proportion of cases caused by the delta variant was $20 \%$ overall, this increased to $74 \%$ of new sequence cases in the week starting 31 May 2021.

The analysis found that 196 (2.3\%) patients with the delta variant compared with $764(2.2 \%)$ patients with the alpha variant were admitted to hospital within 14 days, giving an adjusted hazard ratio of 2.26 (95\% confidence interval 1.32 to 3.89).

The risk of attending hospital for emergency care or being admitted to hospital within 14 days of infection with the delta variant was also one and a half times greater compared with the alpha variant (adjusted hazard ratio $1.45,95 \% \mathrm{CI} 1.08$ to 1.95$)$. Most patients were unvaccinated (74\%) across both groups.

One limitation of the study is that the authors had no access to information about patients' comorbidities, which are known to contribute to hospital admission risk. The study instead indirectly accounted for this using age, sex, ethnicity, and deprivation levels. Another limitation is that some demographics may have been more likely to seek hospital care, which could have biased the results. Also, there may be possible bias on which samples were selected to have whole genome sequencing performed.

The study was carried out by researchers from Public Health England and Cambridge University and was funded by UK Research and Innovation, the Medical Research Council, the UK Department of Health and Social Care, and the National Institute for Health Research.

Anne Presanis, one of the study's lead authors and senior statistician at the MRC Biostatistics Unit, University of Cambridge, said, “Our analysis highlights that in the absence of vaccination, any delta outbreaks will impose a greater burden on healthcare than an alpha epidemic."

A preliminary study from Scotland previously reported a doubling in risk of hospital admission with the delta variant compared with the alpha variant. This used patients' initial polymerase chain reaction test results and determined which variant they had by testing for a specific gene that is more common in the delta variant. ${ }^{2}$

1 Twohig KA, Nyberg T, Zaidi A, etalCOVID-19 Genomics UK (COG-UK) consortium. Hospital admission and emergency care attendance risk for SARS-CoV-2 delta (B.1.617.2) compared with alpha (B.1.1.7) variants of concern: a cohort study. Lancet Infect Dis 2021;S1473-3099(21)00475-8. pmid: 34461056

2 Sheikh A, McMenamin J, Taylor B, Robertson CPublic Health Scotland and the EAVE II Collaborators. SARS-CoV-2 Delta VOC in Scotland: demographics, risk of hospital admission, and vaccine effectiveness. Lancet 2021;397:2461-2. doi: 10.1016/S0140-6736(21)01358-1. pmid: 34139198 terms and conditions for the duration of the covid-19 pandemic or until otherwise determined by BMJ. You may use, download and print the article for any lawful, non-commercial purpose (including text and data mining) provided that all copyright notices and trade marks are retained.
This article is made freely available for use in accordance with BMJ's website 